\title{
Osteogenic response under the periosteum by magnesium implantation in rat tibia
}

\author{
Kenji ODASHIMA ${ }^{1}$, Yoshinaka SHIMIZU², Yuya SANO², Akiko YAMAMOTO ${ }^{3}$, Toshiji MUKAI ${ }^{4}$, Yukyo TAKADA ${ }^{5}$, \\ Yuta YANAGISAWA ${ }^{1}$, Yoshimichi IMAI ${ }^{6}$, Tetsu TAKAHASHI ${ }^{1}$ and Hiroyuki KUMAMOTO ${ }^{2}$ \\ ${ }^{1}$ Division of Oral and Maxillofacial Surgery, Department of Oral and Medicine and Surgery, Graduate School of Dentistry, Tohoku University, 4-1 \\ Seiryo-machi, Aobaku, Sendai, 980-8575, Japan \\ ${ }^{2}$ Division of Oral Pathology, Department of Oral Medicine and Surgery, Graduate School of Dentistry, Tohoku University, 4-1 Seiryo-machi, Aobaku, \\ Sendai, 980-8575, Japan \\ ${ }^{3}$ Biometals Group, Biomaterials Unit, Nano-life Field, International Center for Materials Nanoarchitectonics, National Institute for Materials Science, \\ 1-1 Namiki, Tsukuba, Ibaraki, 305-0044, Japan \\ ${ }^{4}$ Department of Mechanical Engineering, Kobe University, 1-1 Rokkadai-cho, Nada-ku, Kobe, 657-8501, Japan \\ ${ }^{5}$ Division of Dental Biomaterials, Tohoku University Graduate School of Dentistry, Tohoku University, 4-1 Seiryo-machi, Aobaku, Sendai, 980-8575, \\ Japan \\ ${ }^{6}$ Department of Plastic and Reconstructive Surgery, Tohoku University Graduate School of Medicine, 2-1 Seiryo-machi, Aoba-ku, Sendai, 980-8575, \\ Japan \\ Corresponding author, Yoshinaka SHIMIZU; E-mail: shimizu@dent.tohoku.ac.jp
}

\begin{abstract}
This study was designed to examine osteoconductive effects of $\mathrm{Mg}$ in rats tibia. The animals were sacrificed after 1, 2, and 8 weeks. The elemental analysis was performed using SEM/EDX at week 1. Following X-ray micrography at weeks 2 and 8 , samples were embedded in paraffin. The expression of osteocalcin was observed by immunohistochemical staining. The element concentrations of fibrous capsules around the specimens were also measured by ICP-MS. The concentrations of Ca and P on the surface of the $\mathrm{Mg}$ specimen increased in SEM/EDX. The tissue specimen showed new bone formation on the bone surface near the implanted area. The concentrations of $\mathrm{Mg}, \mathrm{Ca}$, and $\mathrm{P}$ were high in the fibrous capsules surrounding $\mathrm{Mg}$. Implantation induced differentiation of osteoblasts, and this process was considered to be associated with new bone formation. Induction of cell differentiation may be influenced by corrosion products in addition to corroding magnesium.
\end{abstract}

Keywords: Biodegradation, Osteogenic, Magnesium, ICP-MS

\section{INTRODUCTION}

Metals, ceramics, and synthetic polymers are used as medical biomaterials. Titanium alloys and stainlesssteel $\mathrm{Co}-\mathrm{Cr}$ among metal materials have already been widely used in clinical applications as osteosynthesis materials ${ }^{1)}$ in orthopedic surgeries and cardiovascular stents $^{2,3}$ in cardiovascular treatments.

Metallic materials have advantages in mechanical strength, but they also have some problems; remaining inside the body for a long time, metallic materials are known to increase the risk of infection ${ }^{4}$. There is also a concern that ions and small fragments, which are formed from metal materials as partial components due to their corrosion and abrasion, may adversely affect the body ${ }^{5}$. The removal of the materials after healing is therefore necessary, and secondary surgery burdens patients and prolongs the period of rehabilitation. In contrast, polyglycolic acid and poly-L-lactic acid devices, which are synthetic polymer materials, are clinically applied as bioabsorbable materials for osteosynthesis devices and sutures ${ }^{6,7}$. Since those materials are decomposed into harmless substances and absorbed in vivo, secondary surgery is unnecessary, and the

Color figures can be viewed in the online issue, which is available at J-STAGE.

Received Jan 26, 2020: Accepted Jun 15, 2020

doi:10.4012/dmj.2020-011 JOI JST.JSTAGE/dmj/2020-011 risk of infection and exposure is low ${ }^{6,7)}$. However, the insufficient mechanical strength of those materials have limited their application, being currently considered problematic $^{8}$. The recent successful improvement of the mechanical strength of the materials have consequently prolonged the absorption period, with the potential for granulation tissue formation and infections ${ }^{9}$.

Applications of magnesium and magnesium alloys for fracture fixation have attracted attention. Magnesium and magnesium alloys have advantages including their biodegradation properties and high mechanical strength ${ }^{10)}$, being suitable for bone tissue regeneration applications. Magnesium, an essential element for the human body, has the two advantages mentioned above, so that a magnesium alloy has received major attraction as a new material to be applied for bone fixation ${ }^{11}$ and vascular stenting ${ }^{12)}$.

Magnesium as a biomaterial has been confirmed to promote new bone formation in the surrounding tissues. In vivo and in vitro examinations have recently reported the important benefit of an osteosynthesis material; magnesium alloys have osteogenic ability ${ }^{13-16)}$. In the fracture model using rabbit ulna in vivo by Chaya et al. ${ }^{177}$, the use of magnesium alloy plate and screw promoted new bone formation and efficient fracture healing until the plate was absorbed. Juan et al. ${ }^{18)}$ reported in vitro 
examination that fluid extraction of magnesium alloy could directly stimulate pre-osteoblasts and induce osteoblast differentiation. In his vivo study, Zhang et al. ${ }^{19)}$ described that a pure $\mathrm{Mg}$ intramedullary nail in the rat femur activated the peripheral nerve in the periosteum, promoting CGRP-mediated osteogenesis. At present, some in vivo studies have reported that corroding magnesium directly influences osteoblasts ${ }^{14)}$; however, the direct effect of corroding magnesium on bone formation have not been showed in vivo studies.

In this study, a magnesium plate was implanted under the tibial periosteum of the rat for morphological, histological and engineering examinations. The concentrations of magnesium, phosphate, and calcium in the fibrous capsule surrounding the magnesium alloy were measured. This study therefore aims to confirm that corroding magnesium can directly induce osteogenesis in the periosteum tissues in the proximity of the implanted magnesium alloy.

\section{MATERIALS AND METHODS}

\section{Experimental materials}

AZ31 magnesium alloy plates (96\% Mg, 3\% Al, 1\% Zn; percentage by mass) (Nippon Kinzoku, Tokyo), with dimensions of $1.0 \times 1.0 \times 0.1 \mathrm{~mm}$, were used in this study ${ }^{20)}$. The surface analysis of the magnesium alloy using energy dispersive X-ray analysis (EDX) was performed to confirm that the alloy has no impurities. A pure titanium plate (TR270C-H, Takeuchi Kinzokuhakufun Kogyo, Tokyo) with the same size and thickness as that of magnesium was used to serve as a control. The surface of the plate used was mechanically polished to ensure good surface properties. Immediately before implantation, the magnesium alloy plate was immersed in a $10 \%$ acetic acid solution for about $60 \mathrm{~s}$ in order to remove oxide film, washed with acetone to remove the oil on the surface, and used after drying. In order to prevent its corrosion due to contact with different metals, the magnesium alloy plate was gripped with a resin claw. Titanium was washed with acetone and used after drying.

\section{Experimental animals}

All experiments involving animals were approved by the Tohoku University Environment and Safety Committee Animal Experiment Committee (2015DnA-033) and conducted based on Regulations for animal experiments and Related Activities at Tohoku University (Sendai, Japan). Forty-two male Wistar rats (average weight: 338.9 g) aged approximately 18 weeks were grouped as shown in Table 1, and observed 1, 2, and 8 weeks after surgery (week 1, 2, and 8). For micro-CT image and histological observation, same rats (total 10 rats) were used in sham and titanium groups, respectively in right and left hind limb, at 2 and 8 weeks. Ten rats were implanted magnesium plates in right hind limb at 2 and 8 weeks. For SEM/EDX observation, both hind limbs of two rats were used. The rats were housed one or two per cage with food and water ad libitum.

\section{Surgery procedure}

Surgery procedure was performed under general anesthesia by inhalation of $5 \%$ isoflurane (Isoflurane for Animal, Intervet, Tokyo, Japan). Local anesthesia with $2 \%$ lidocaine containing epinephrine (Astra Zeneca, Osaka, Japan) was injected into animals after their hind legs were shaved. Skin incision was made to indicate the subcutaneous connective tissue, and then a transverse incision was performed under the periosteum, showing the bone surface. A magnesium alloy plate (the magnesium group) and a pure titanium plate (the titanium group) were placed beneath the

Table 1 Experimental animal groups

\begin{tabular}{|c|c|c|c|c|}
\hline Group & Implant & $\mathrm{n}$ & Observation period & $\mathrm{n}$ \\
\hline \multirow{5}{*}{$\mu \mathrm{CT}$} & Sham & 10 & $\begin{array}{l}2 w \\
8 w\end{array}$ & $\begin{array}{l}5 \\
5\end{array}$ \\
\hline & \multirow{2}{*}{$\mathrm{Ti}$} & \multirow{2}{*}{10} & $2 \mathrm{w}$ & 5 \\
\hline & & & $8 w$ & 5 \\
\hline & \multirow{2}{*}{$\mathrm{Mg}$} & \multirow{2}{*}{10} & $2 \mathrm{w}$ & 5 \\
\hline & & & $8 w$ & 5 \\
\hline \multirow{2}{*}{ SEM/EDX } & $\mathrm{Ti}$ & 2 & $1 \mathrm{w}$ & 2 \\
\hline & $\mathrm{Mg}$ & 2 & $1 \mathrm{w}$ & 2 \\
\hline \multirow{6}{*}{ Histopathology } & \multirow{2}{*}{ Sham } & \multirow{2}{*}{10} & $2 \mathrm{w}$ & 5 \\
\hline & & & $8 w$ & 5 \\
\hline & \multirow{2}{*}{$\mathrm{Ti}$} & \multirow{2}{*}{10} & $2 \mathrm{w}$ & 5 \\
\hline & & & $8 w$ & 5 \\
\hline & \multirow{2}{*}{$\mathrm{Mg}$} & \multirow{2}{*}{10} & $2 \mathrm{w}$ & 5 \\
\hline & & & $8 w$ & 5 \\
\hline \multirow{4}{*}{ ICP-MS } & Sham & 5 & $8 w$ & 5 \\
\hline & $\mathrm{Ti}$ & 5 & $8 w$ & 5 \\
\hline & \multirow{2}{*}{$\mathrm{Mg}$} & \multirow{2}{*}{10} & $2 w$ & 5 \\
\hline & & & $8 w$ & 5 \\
\hline
\end{tabular}




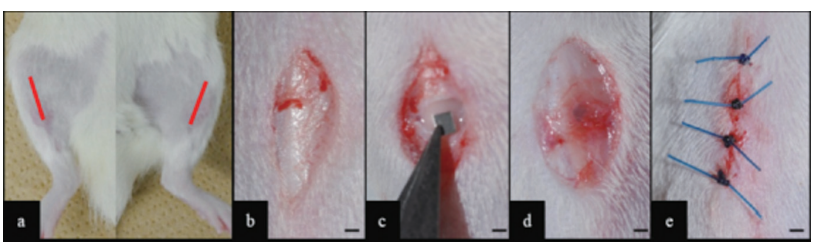

Fig. 1 Schematic representation of animal models. (a) Skin incision line. (b) Subperiosteal incision line. (c) Surgical implantation of a metal plate by ceramic tweezers. (d) A plate under the periosteum. (e) Sutures. Scale bars $=1.0 \mathrm{~mm}$

periosteum, and finally, the incisions were sutured. In the control group, only the periosteum was detached by the same procedure as used in the magnesium and titanium groups, and the incisions were sutured (the sham group) (Fig. 1).

\section{Macroscopic findings}

In the three groups, the mice were received intraperitoneal injection of pentobarbital and sacrificed under the excessive anesthesia 1,2 , and 8 weeks after surgery. As shown in Table 1, the implanted plate surfaces were analyzed using scanning electron microscopy with energy dispersive X-ray spectroscopy (SEM/ EDX) one week after surgery. Two and 8 weeks after surgery, the wounds were macroscopically observed and then fixed by perfusing $4 \%$ paraformaldehyde (PFA) from the heart and abdominal aorta. The specimen were prepared for histological observation.

\section{Micro X-ray CT observation}

The implanted area in the tibia was cut off in order to capture the enlarged image as much as possible with micro CT. The obtained samples were observed using micro X-ray CT (ScanXmate-E090, Comscantecno, Kanagawa, Japan). The scanning conditions were as follows: tube voltage, $87 \mathrm{kV}$; electrical current, $98 \mu \mathrm{A}$; and image pixel size, $0.05 \mu \mathrm{m}$. The imaging data was observed on a horizontal section.

\section{SEM/EDX analysis}

Prior to implantation, the surfaces of the magnesium and titanium plates were examined using SEM/EDX analysis (JSM-6390LA; JEOL, Tokyo, Japan). The same analysis was performed to observe precipitates and corrosion products on the surface of magnesium and titanium plates one week after implantation. The scanning conditions were as follows: $10 \mathrm{kV}, 1.0 \mathrm{nA}$, and $10 \mathrm{~mm}$ working distance.

\section{Histological preparation}

After photographing of micro X-ray CT, the obtained samples were refixed with $4 \%$ PFA at $3^{\circ} \mathrm{C}$, and decalcified at $3^{\circ} \mathrm{C}$ with $10 \%$ ethylenediaminetetraacetate acid (EDTA) solution (OSTEOSOFT ${ }^{\circledR}$ Merck KGaA, Darmstadt, Germany) for approximately 2 weeks. Subsequently, the titanium and magnesium plates were carefully removed. The implanted area including the tibia and surrounding tissues were horizontally sectioned and detached to be embedded in paraffin according to a conventional method. Serial sections with a thickness of approximately $4 \mu \mathrm{m}$ were prepared.

\section{Tissue staining and morphometry}

Using hematoxylin and eosin (HE) staining (Mayer's Hematoxylin Solution WAKO, Tokyo, Japan), morphological observation and morphometry of new bone was performed to confirm pathological reactions around the materials. The images of new bones on the cortical bone surfaces around the titanium and magnesium plates, segmented by Image J software ${ }^{21)}$, were measured to analyze the amount of new bone formation.

\section{Immunohistological staining}

To confirm osteogenesis around the implanted material, immunohistochemical staining of osteocalcin was performed. Anti-Osteocalcin antibody (ab13420 abcam, Tokyo, Japan) was used as a primary antibody. The sections were deparaffinized, hydrated with distilled water, and washed with a phosphate buffer solution. In order to activate the antigen, it was left in a $0.5 \%$ trypsin solution at $37^{\circ} \mathrm{C}$ for $20 \mathrm{~min}$. The specimens were placed in a bovine serum albumin solution (ab64234 Abcam) for $30 \mathrm{~min}$ at room temperature to inhibit nonspecific binding of proteins. The specimens were reacted with the primary antibody (200-fold dilution) at $4^{\circ} \mathrm{C}$ overnight. The tissue preparations were then washed with sterile distilled water, followed by incubation with biotinylated goat anti-rabbit IgG secondary antibodies (ab97040 Abcam) and staining with a 3,3'-diaminobenzidine solution. Namely, a streptavidin-biotin method was used for antibody detection. Nuclei were counterstained with Mayer hematoxylin. Osteocalcin was quantitatively measured based on measurement methods introduced by Pedrosa et $a l .{ }^{22)}$ and Esteves et al. ${ }^{23)}$. The samples were grouped into the following 4 groups according to staining scores: negative (-), 1 point; positive $(+), 2$ points; super positive (++), 3 points; and hyper positive labels $(+++)$, 4 points. Statistical analysis was performed for each material and week individually.

Quantitative analysis of local (capsules around materials) elements by inductively coupled plasma mass spectrometry (ICP-MS)

Rats in the three groups were sacrificed 2 or 8 weeks after surgery, and then the fibrous capsules around the implanted materials were sectioned. After weighing the sectioned samples, they were placed in $3 \mathrm{~mL}$ of nitric acid and digested at $170^{\circ} \mathrm{C}$ using a microwave digester. After 5 min of treatment, the samples were degraded at $200^{\circ} \mathrm{C}$ for $45 \mathrm{~min}$. The concentrations per unit weight of elements in the solution such as magnesium, calcium, and phosphorus were quantified using ICP-MS (Agilent 8800, Agilent, Tokyo, Japan). The concentrations of magnesium, calcium, and phosphorus in the sham and titanium implantation groups were analyzed 8 weeks after implantation, and that in the magnesium group was 
statistically analyzed 2 and 8 weeks after implantation (Table 1).

\section{Statistical analysis}

Statistical analysis used the two-way analysis of variance and multiple test (the Tukey test). The software used was StatMate V (Atomus, Oita, Japan). $p$ Values of less than 0.05 were considered to indicate statistical significance.

\section{RESULTS}

Macroscopic findings in animal experiments (Fig. 2)

During the study period, the rats showed neither loss of appetite nor abnormal behavior. No apparent weight loss was observed. Wound healing was macroscopically good in the three groups at all weeks, and no obvious infectious findings such as redness or swelling was observed on the skin at the implant insertion sites. There was no difference in macroscopic findings of wound healing between the groups.

\section{SEM/EDX findings (Figs. 3-6)}

The surface analyses of the magnesium alloy and titanium before implantation are shown in Figs. from 3 to 6 . In the magnesium alloy plate, magnesium and a smallamount of Al were detected (Fig. 3). Titanium plate consisted of only $\mathrm{Ti}$, and the structure was homogeneous (Fig. 4).

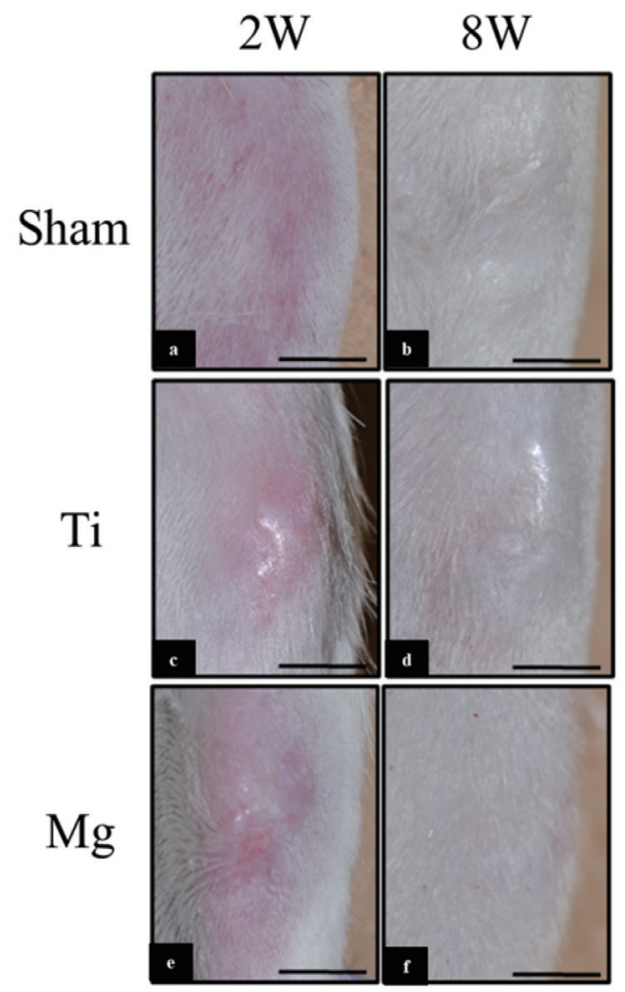

Fig. 2 Macroscopic findings (the 3 groups 2 and 8 weeks after surgery).

(a) and (b) Sham procedure. (c) and (d) Pure titanium. (e)and (f) Magnesium alloy. Scale bars $=3.0 \mathrm{~mm}$ (a)

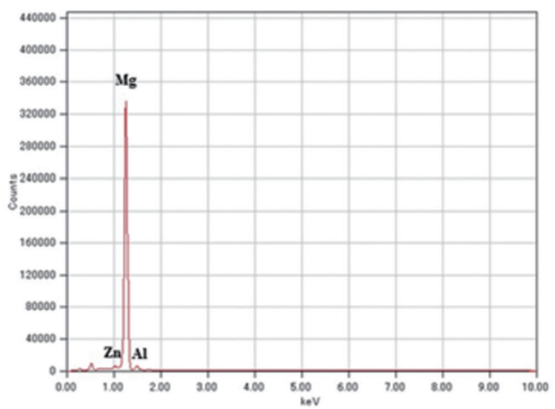

(b)

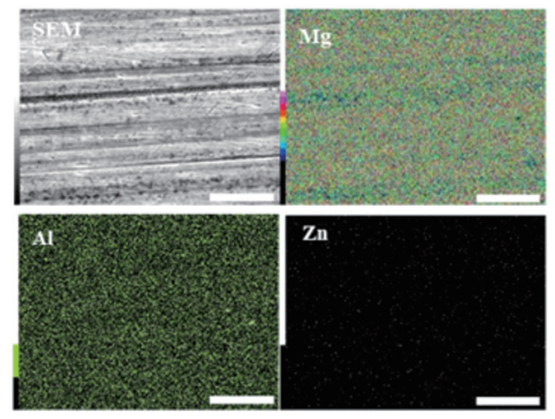

Fig. 3 Scanning electron microscopy (SEM) images and energy dispersive X-ray spectroscopy (EDX) analysis of magnesium alloy plate surface morphology before implantation.

(a) EDX analysis. (b) EDX mapping. Scale bars $=25$ $\mu \mathrm{m}$

(a)

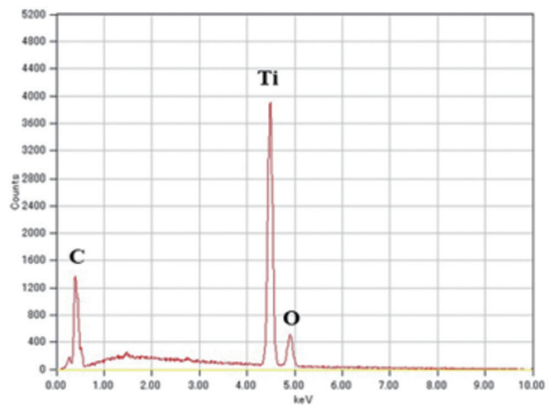

(b)
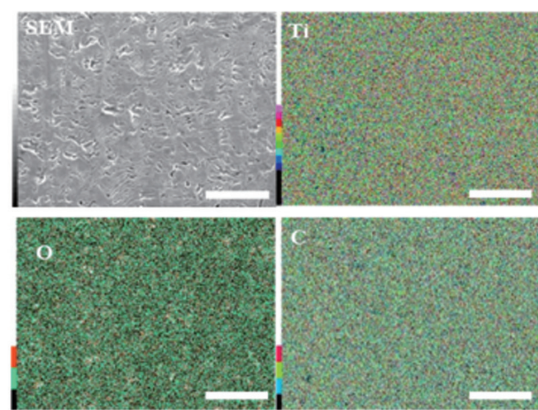

Fig. 4 Scanning electron microscopy (SEM) images and energy dispersive X-ray spectroscopy (EDX) analysis of titanium plate surface morphology before implantation.

(a) EDX analysis. (b) EDX mapping. Scale bars $=25$ $\mu \mathrm{m}$ 
(a)
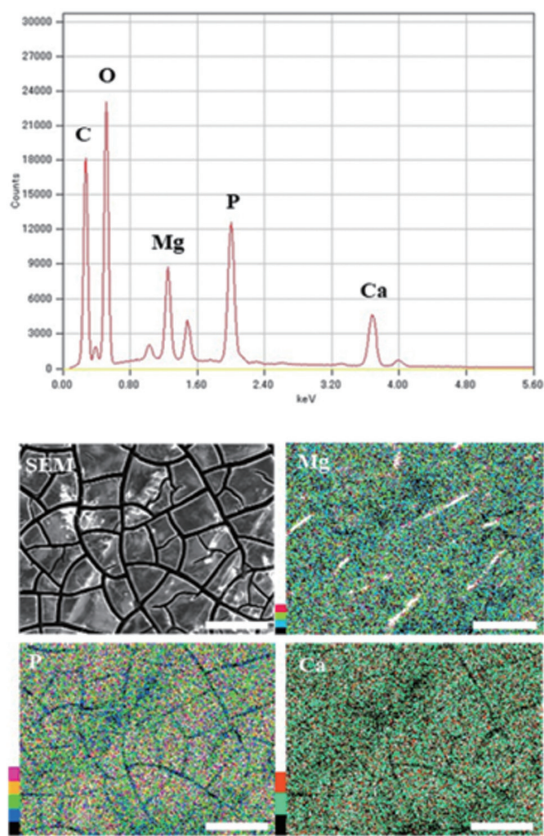

(b)

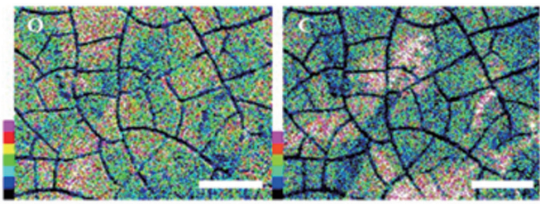

Fig. 5 Scanning electron microscopy (SEM) images and energy dispersive X-ray spectroscopy (EDX) analysis of magnesium alloy plate surface morphology one week after implantation.

(a) EDX analysis. (b) EDX mapping. Scale bars $=25$ $\mu \mathrm{m}$

The surface of the magnesium plate at week 1 was rough as shown in Fig. 5 and showed deposits including $\mathrm{P}, \mathrm{Ca}, \mathrm{O}$, and $\mathrm{C}$. A small amount of $\mathrm{Mg}$ was deposited on the surface of the magnesium plate (Fig. 5). As shown in Fig. 6, a large amount of $\mathrm{C}$ and $\mathrm{O}$ were observed on the surface of the pure titanium plate at week 1 , but no apparent amount of $\mathrm{P}$ and Ca was observed (Fig. 6).

\section{Micro X-ray CT findings (Fig. 7)}

Horizontal cross-sectional images including samples of the three groups are shown in Fig. 7. In the sham group, no obvious new bone formation was observed on the bone surface near the implanted area at week 2 or 8. In the magnesium group, new bone formation was observed around magnesium at both weeks 2 and 8, and the new bone surrounded the side of the magnesium plate at week 8 . In the titanium group, a rough surface was observed around titanium at week 2 , but no obvious new bone was observed at week 8 .

Histological findings of surrounding tissues (Fig. 8) Figure 8 shows histological findings of the implanted area. Hematoxylin and eosin stained specimens showed (a)

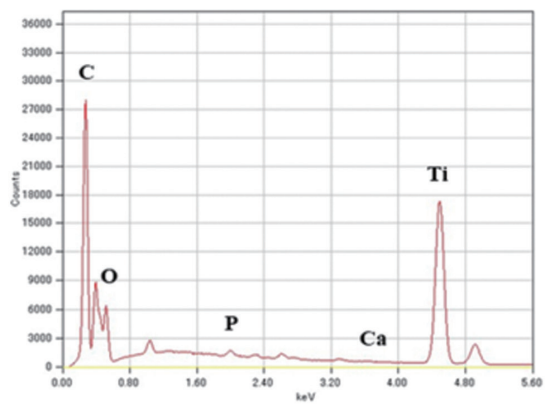

(b)
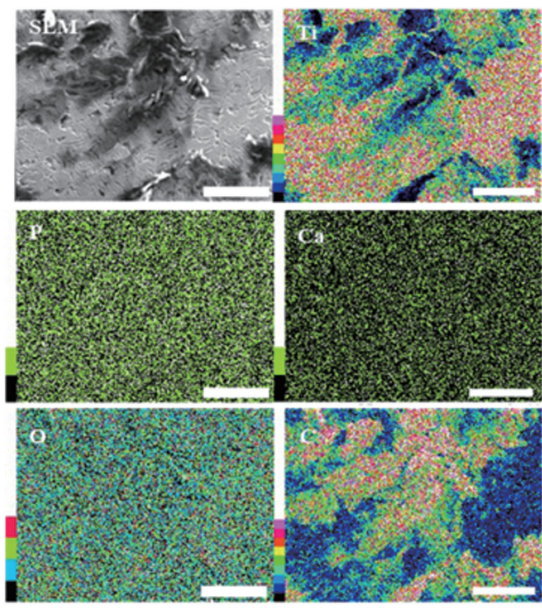

Fig. 6 Scanning electron microscopy (SEM) images and energy dispersive X-ray spectroscopy (EDX) analysis of titanium plate surface morphology one week after implantation.

(a) EDX analysis. (b) EDX mapping. Scale bars $=25$ $\mu \mathrm{m}$

capsules of granulation tissue and fibrous connective tissue around the implanted materials in the magnesium and titanium groups. In the sham group, a granulationlike fibrous tissue was observed in the upper part of the periosteum. In all three groups, mild inflammatory cell infiltration was observed at week 2 , and fibrous tissues with rich collagen fibers were observed at week 8 . In the magnesium implant group, no obvious gas cavities formation was observed around the implanted area.

New bone morphometry with tissue specimens (Figs. 9 and 10)

The amount of new bone on the surface of the tibia under the implanted samples was observed and measured. In the sham and titanium groups, no obvious new bone formation was observed at week 2 or 8 . In the magnesium group, irregular shaped new bone was observed on the bone surface in the implanted area (Fig. 9). In the three groups, the amount of new bone increased from week 2 to week 8 . In both the 2 and 8 weeks, the magnesium group had apparently more new bones $(p<0.05)$ (Fig. 10). 


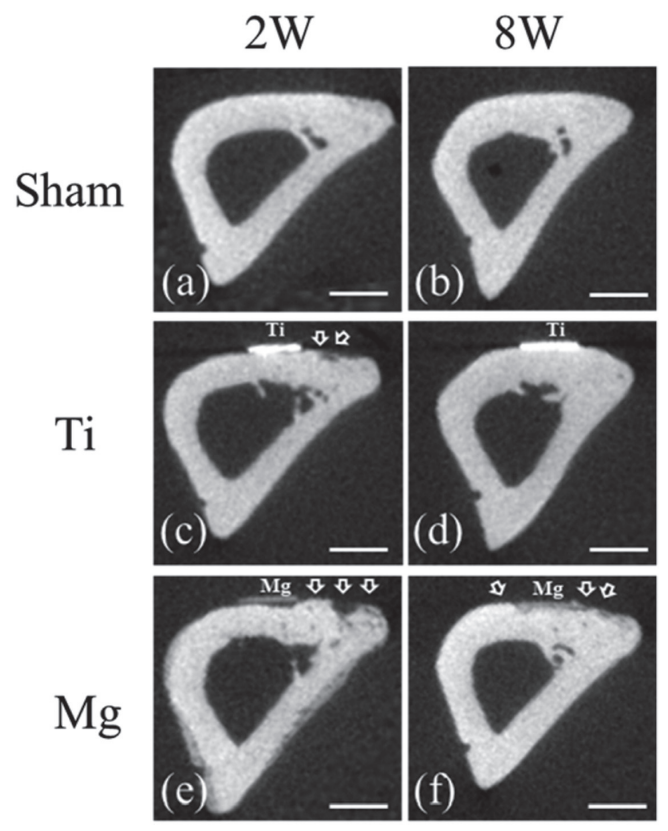

Fig. 7 Micro-computed tomography ( $\mu \mathrm{CT}$ ) 2-dimensional images of implantation materials on the tibia and sham procedure 2 and 8 weeks after implantation. The arrows indicate areas of osteogenesis. (a) and (b) Sham procedure. (c) and (d) Pure titanium. (e) and (f) Magnesium alloy. Scale bars $=1.0 \mathrm{~mm}$

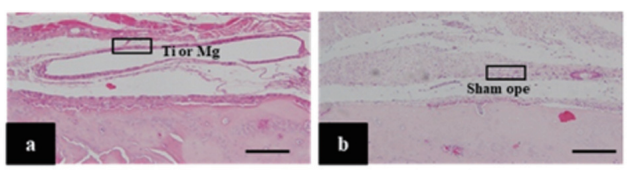

$2 \mathrm{~W}$

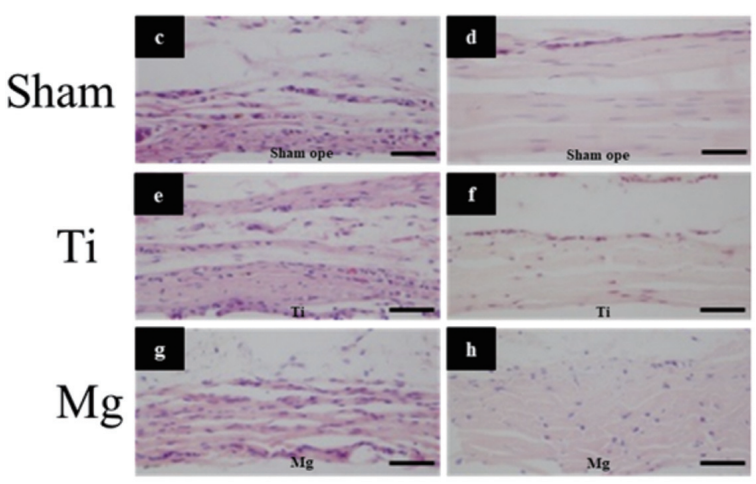

Fig. 8 Histological findings of surrounding tissue in operative site.

(a) The magnesium and titanium groups. The rectangle indicates the area observed in $\mathrm{e}-\mathrm{h}$. (b) The sham group. The rectangle indicates the area observed in c and d. Scale bars $=200 \mu \mathrm{m}$. (c) and (d) Granulation tissues and fibrous tissues surrounding sham procedure area at weeks 2 and 8 . Scale bars $=50 \mu \mathrm{m}$. (e), (f), (g), and (h) Granulation tissues and fibrous tissues surrounding metal plates at weeks 2 and 8 . Scale bars $=50 \mu \mathrm{m}$
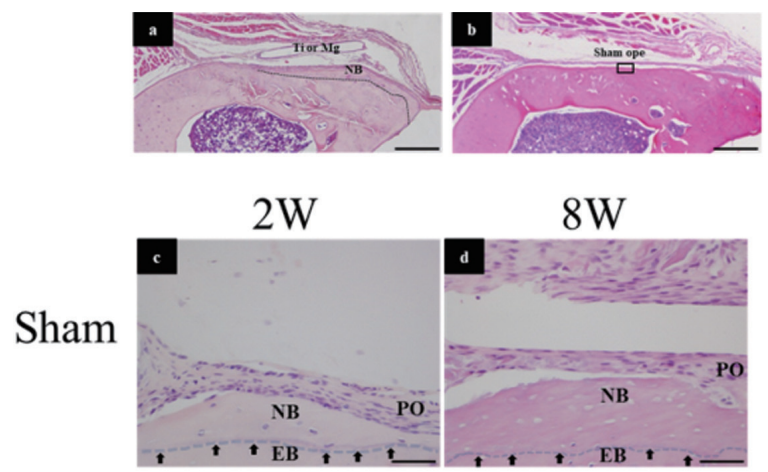

$\mathrm{Ti}$
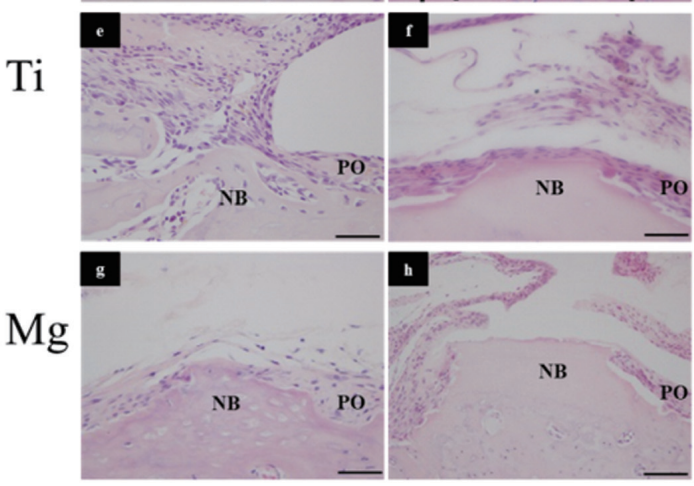

Fig. 9 Histopathological findings of hematoxylin and eosin (H\&E).

(a) and (b) Osteogenesis region under the metal plates or sham procedure. Scale bars $=500 \mu \mathrm{m}$. (c), (d), (e), (f), (g), and (h) Osteogenesis region under the metal plates or sham procedure 2 and 8 weeks after implantation or sham procedure. The arrows indicate border line between EB and NB. NB: new bone, Bone: existing bone, and PO: periosteum.

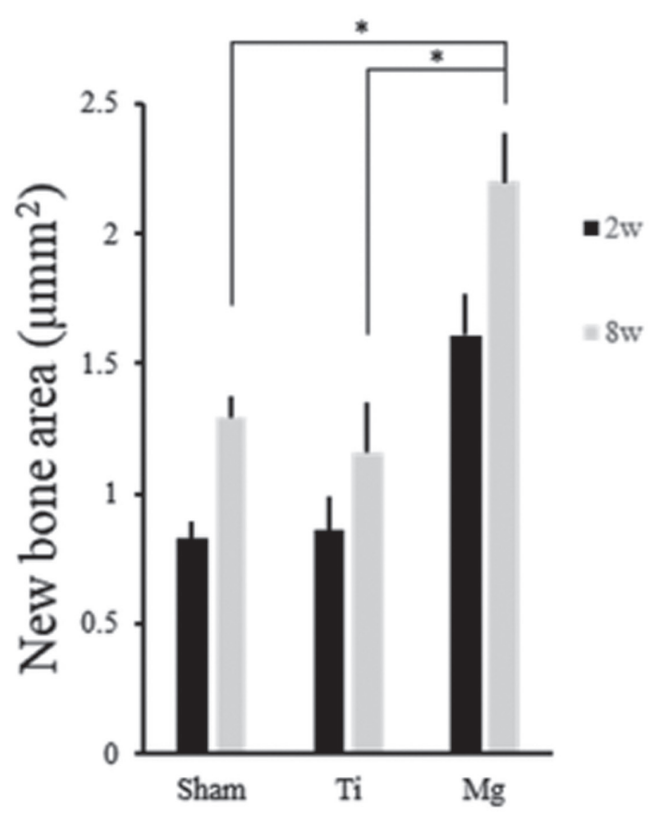

Fig. 10 Quantitative analysis of H\&E stained new bone areas in the 3 groups at weeks 2 and $8 .{ }^{*} p<0.05$ 
Immunohistochemical study (Figs. 11 and 12)

Immunohistochemical study confirmed the expression of osteocalcin in cells on the cortical bone surface corresponding to the periosteal region in the magnesium and titanium groups (Fig. 11). A quantitative analysis

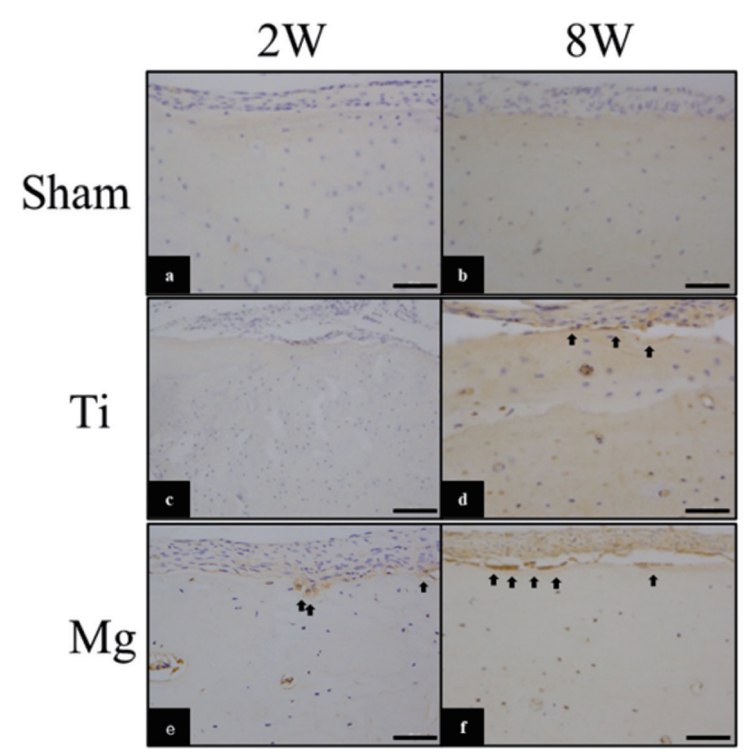

Fig. 11 Immunohistochemical staining findings of osteocalcin (OC) on osteogenesis region under the metal plates or sham procedure 2 and 8 weeks after implantation.

The arrows indicate OC positive cells Scale bars $=50 \mu \mathrm{m}$

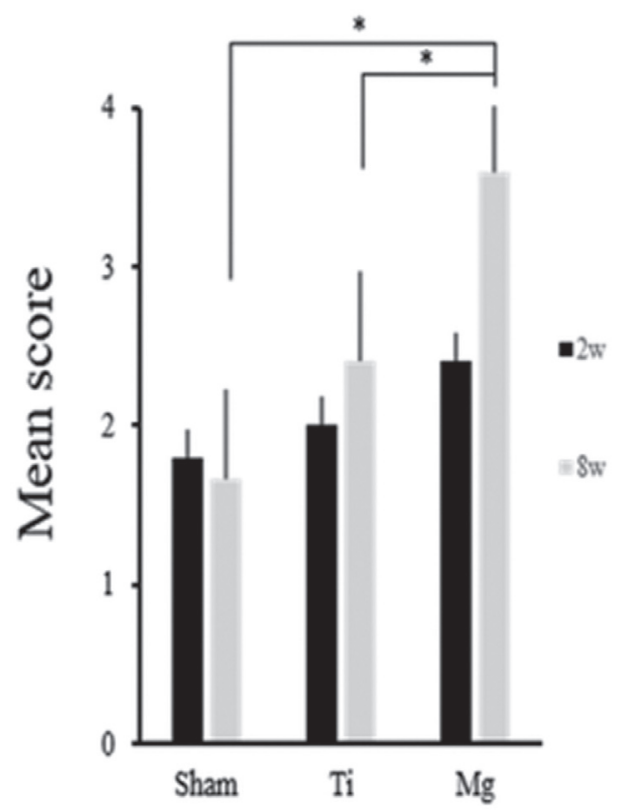

Fig. 12 Immunohistochemical analysis of osteocalcin (OC) during bone regeneration process in the 3 groups at weeks 2 and 8 .

${ }^{*} p<0.05$ of osteocalcin is shown in Fig. 12. The expression of osteocalcin tended to increase over time in the magnesium and titanium groups. At week 8, the sham group showed a decrease in the expression of osteocalcin, while the magnesium group showed a significantly higher expression of osteocalcin compared with the titanium and sham groups (Fig. 12).

\section{Quantitative analysis by ICP-MS (Figs. 13 and 14)}

Magnesium concentration in the magnesium group at week 8 was significantly higher than that in the sham group at week $8(p<0.05)$. Magnesium concentration in

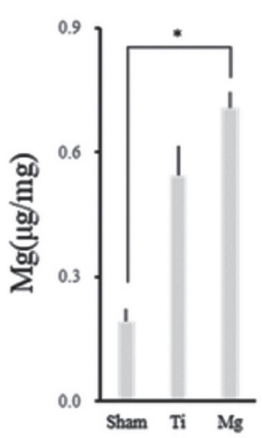

(a)

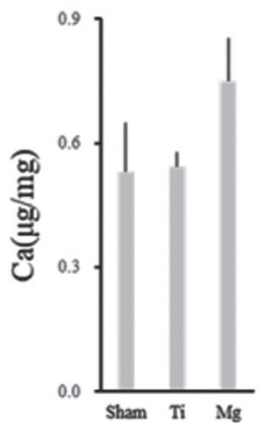

(b)

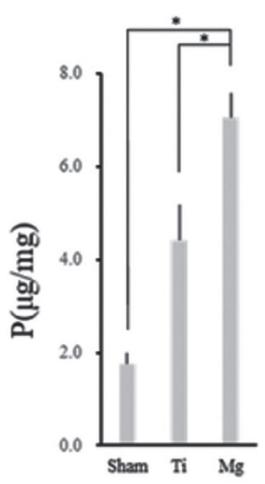

(c)
Fig. 13 Quantitative analyses of $\mathrm{Mg}, \mathrm{Ca}$, and $\mathrm{P}$ concentrations in the fibrous capsules surrounding the two metals and sham operation area by Inductively Coupled Plasma Mass Spectrometry (ICP-MS).

(a), (b) and (c) $\mathrm{Mg}, \mathrm{Ca}$, and $\mathrm{P}$ concentration of surrounding tissues in the 3 groups 8 weeks after implantation or sham procedure. ${ }^{*} p<0.05$

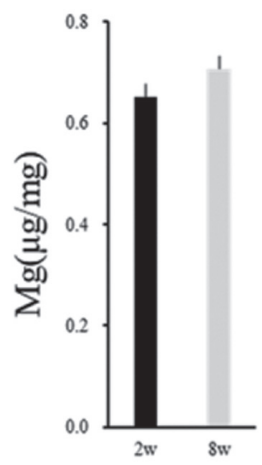

(a)

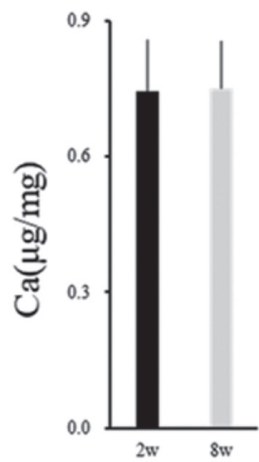

(b)

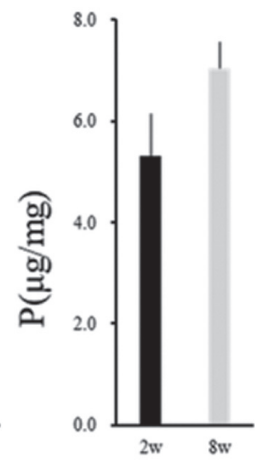

(c)
Fig. 14 Quantitative analyses of $\mathrm{Mg}, \mathrm{Ca}$, and $\mathrm{P}$ concentrations in the fibrous capsules surrounding the magnesium alloy plates by Inductively Coupled Plasma Mass Spectrometry (ICP-MS).

(a) (b) and (c) $\mathrm{Mg}, \mathrm{Ca}$, and $\mathrm{P}$ concentrations of surrounding tissues 2 and 8 weeks after implantation. ${ }^{*} p<0.05$ 
the titanium group at week 8 was higher than that in sham group without significant difference. The calcium concentration in magnesium group at week 8 was the highest among the three groups, but no significant difference was observed. The phosphorus concentration in the magnesium group was significantly higher than that in the sham and titanium groups $(p<0.05)$ (Fig. 13).

In the magnesium group, the magnesium and calcium concentrations were similar at weeks 2 and 8, with no significant difference. The phosphorus concentration at week 8 was higher than that at week 2 in the magnesium group, but no significant difference was observed (Fig 14).

\section{DISCUSSION}

The application of magnesium and magnesium alloys as bone repair implants has been extensively investigated $^{24)}$. No harmful effects of magnesium alloys have been reported ${ }^{25)}$. Huehnerschulte et al. have reported in vivo experiments that showed bone resorption on its surface in the proximity of implanted magnesium ${ }^{11)}$; this resorption, if it is extensive, can be problematic. In this experiment, bone resorption was not seen. Implantation of biomaterials causes local tissue injury, inducing inflammatory reactions. It is necessary to consider a foreign body response when using a biomaterial because it is an exogenous foreign material. In particular, abnormal bone resorption due to the induction of osteoclasts ${ }^{11)}$ should be considered. The influence of magnesium alloy degradation such as eluted ions and generated gas should also be considered. However, this experiment showed no abnormal bone resorption in the sham and titanium groups, and, instead, confirmed that magnesium had little harmful effect on tissues surrounding implanted materials.

Magnesium alloys shows the precipitation of corrosion products during in vivo degradation ${ }^{26)}$. Immediately after forming magnesium oxide, magnesium alloys react with hydroxide ions, carbonate ions, and phosphate ions in the body to precipitate magnesium hydroxide, magnesium carbonate, and magnesium phosphate in degradation. At the same time, calcium ions in the body react to precipitate calcium hydroxide, calcium carbonate, and calcium phosphate ${ }^{13,14,27-29)}$. In this experiment, calcium-phosphorus precipitation was also observed on the surface layer of the magnesium alloy, and the precipitation of calcium hydroxide and calcium phosphate was observed in addition to magnesium hydroxide and magnesium phosphate from week 2 . Since calcium phosphate has been used as a bone graft material showing osteoconduction ${ }^{30)}$, the precipitation of calcium phosphate was likely to be the reason for the high bone affinity of magnesium alloys.

In the tissues surrounding the magnesium alloy, the concentrations of calcium and phosphorus as well as magnesium that was thought to have precipitated during degradation were also high. Calcium-sensing receptors are expressed in osteoblasts and osteoclasts to regulate calcification and bone metabolism by remodeling the surrounding bone according to the extracellular calcium concentration $^{31)}$. In the magnesium group, the induction of calcium and phosphorus in the surrounding tissues may promote the formation of precipitates such as calcium phosphate. Consequently, this induction may be associated with the induction of calcification of new bone around the implanted magnesium alloy. On the other hand, induction of magnesium was also observed around titanium at 8 weeks. Magnesium is induced in wound healing ${ }^{32)}$ and is known to contribute to healing ${ }^{33)}$. The delayed healing by the titanium implantation may induce magnesium ions in the titanium transplant group.

The osteoconductive effects of magnesium has attracted attention before its research and development became prominent ${ }^{34)}$. Retting et al. confirmed the formation of bone around a magnesium implant ${ }^{30)}$; however, the bone formation mechanism of magnesium alloys remains still unclear. Although an experiment in which an intramedullary nail was implanted has recently confirmed bone formation via the sympathetic nervous system, this report does not explain its mechanism of directly forming new bone because magnesium had a distance from the bone formation area. Intrathecal tumors and inflammation are pathologically well known to induce reactive bone on the surface of the cortical bone $^{35)}$, but their direct effects on bone formation are unclear. This experiment confirmed apparent expression of osteocalcin in cells around magnesium. This suggested that corroding magnesium can induce osteoblast differentiation. Bone formation was considered to be sustained because the degradation of the magnesium alloy continued until week $8^{30}$.

Bone formation requires not only the induction of cell differentiation but also the formation of a bone matrix, which occupies most of the bone tissue. The amount of new bone formation has been confirmed to be significantly higher in magnesium than that in titanium $^{18)}$. A condition in which the calcium level is high such as hypercalcemia induces ectopic calcifications in the blood vessel walls ${ }^{36,37)}$. Calcium influx around magnesium may result in increased calcification around a magnesium implant.

The clinical application of magnesium has been actively studied ${ }^{11,12,17,19,38-43)}$, and those studies have shown that magnesium alloys have the property of in vivo degradation while maintaining mechanical strength. Thus, magnesium alloys are expected to be useful as alternative materials in surgical treatments to avoid secondary surgery. Previously, the use of magnesium alloys as fracture fixation plates, screws and fixation screws have been reported; Naujokat et al. have published results of the applications of magnesium alloys even in human ${ }^{42)}$. Understanding the mechanism of magnesium-induced bone formation as described in this study may lead to the development of new devices that contribute to the improvement of healing efficiency in bone treatment. 


\section{CONCLUSION}

The application of magnesium increased the activity of osteoblasts in the periosteum at the implanted area and induced new bone formation. Corroding magnesium and surface corrosion products promote osteoblast differentiation and induction, leading to enhanced new bone formation.

\section{REFERENCES}

1) Geetha M, Singh AK, Asokamani R, Gogia AK. Ti based biomaterials, the ultimate choice for orthopaedic implants: A review. Prog Mater Sci 2009; 54: 397-425.

2) Mani G, Feldman MD, Patel D, Agrawal CM. Coronary stents: A aterials perspective. Biomaterials 2007; 28: 1689-1710

3) O'Brien B, Carroll W. The evolution of cardiovascular stent materials and surfaces in response to clinical drivers: A review. Acta Biomater 2009; 5: 945-958.

4) Thanni LO, Aigoro NO. Surgical site infection complicating internal fixation of ractures: incidence and risk factors. J Natl Med Assoc 2004; 96: 1070-1072.

5) Thomas P, Weik T, Roider G, Summer B, Thomsen M. Influence of surface coating on metal ion release: Evaluation in patients with metal allergy. Orthopedics 2016; 39: S24-30.

6) Suuronen R, Pohjonen T, Wessman L, Törmälä P, Vainionpää S. New generation biodegradable plate for fracture fixation: comparison of bending strengths of mandibular osteotomies fixed with absorbable self-reinforced multi-layer poly-l-lactide plates and metallic plates: an experimental study in sheep. Clin Mater 1992; 9: 77-84.

7) Masuda S, Fujibayashi S, Otsuki B, Kimura H, Neo M, Matsuda S. The dural repair using the combination of polyglycolic acid mesh and fibrin glue and postoperative management in spine surgery. J Orthop Sci 2016; 21: 586590.

8) Ulery BD, Nair LS, Laurencin CT. Biomedical applications of iodegradable polymers. J Polym Sci B Polym Phys 2011; 49: 832-864.

9) Juutilainen T, Hirvensalo E, Partio EK, Rokkanen P. Complications in the first 1043 operations where selfreinforced poly-L-lactide implants were used solely for tissue fixation in orthopaedics and traumatology. Int Orthop 2002; 26: 122-125.

10) Staiger MP, Pietak AM, Huadmai J, Dias G. Magnesium and its alloys as orthopedic biomaterials: a review. Biomaterials [Internet] 2006; 27: 1728-1734.

11) Huehnerschulte TA, Angrisani N, Rittershaus D, Bormann $\mathrm{D}$, Windhagen $\mathrm{H}$, Meyer-Lindenberg A. In vivo corrosion of two novel magnesium alloys ZEK 100 and AX 30 and their mechanical suitability as biodegradable implants. Materials 2011; 4: 1144-1167.

12) Kereiakes DJ, OnumaY, Serruys PW, Stone GW. Bioresorbable vascular scaffolds for coronary revascularization. Circulation 2016; 134: 168-182.

13) Witte F, Kaese V, Haferkamp H, Switzer E, Meyer-Lindenberg $\mathrm{A}$, Wirth CJ, et al. In vivo corrosion of four magnesium alloys and the associated bone response. Biomaterials 2005; 26 : $3557-3563$.

14) Janning C, Willbold E, Vogt C, Nellesen J, Meyer-Lindenberg A, Windhagen $\mathrm{H}$, et al. Magnesium hydroxide temporarily enhancing osteoblast activity and decreasing the osteoclast number in peri-implant bone remodelling. Acta Biomater 2010; 6: 1861-1868.

15) Castellani C, Lindtner RA, Hausbrandt P, Tschegg E, StanzlTschegg SE, Zanoni G, et al. Bone-implant interface strength and osseointegration: Biodegradable magnesium alloy versus standard titanium control. Acta Biomater 2011; 7: 432-440.
16) Rahim MI, Weizbauer A, Evertz F, Hoffmann A, Rohde M, Glasmacher B, et al. Differential magnesium implant corrosion coat formation and contribution to bone bonding. $\mathrm{J}$ Biomed Mater Res A 2017; 105: 697-709.

17) Chaya A, Yoshizawa S, Verdelis K, Myers N, Costello BJ, Chou DT, et al. In vivo study of magnesium plate and screw degradation and bone fracture healing. Acta Biomater 2015; 18: 262-269.

18) Díaz-Tocados JM, Herencia C, Martínez-Moreno JM, Montes de Oca A, Rodríguez-Ortiz ME, Vergara N, et al. Magnesium Chloride promotes Osteogenesis through notch signaling activation and expansion of mesenchymal stem cells. Sci Rep 2017; 7: 7839.

19) Zhang Y, Xu J, Ruan YC, Yu MK, O'Laughlin M, Wise H, et al. Implant-derived magnesium induces local neuronal production of CGRP to improve bone-fracture healing in rats. Nat Med 2016; 22: 1160-1169.

20) Guo J, Zhou Y, Liu C, Wu Q, Chen X, Lu J. Wire arc additive manufacturing of AZ31 magnesium alloy: Grain refinement by adjusting pulse frequency. Mater (Basel, Switzerland) 2016; 9: 760-775.

21) Schneider CA, Rasband WS, Eliceiri KW. NIH Image to ImageJ: 25 years of image analysis. Nat Methods 2012; 9: 671-675.

22) Pedrosa WF, Okamoto R, Faria PEP, Arnez MFM, Xavier SP, Salata LA. Immunohistochemical, tomographic and histological study on onlay bone graft remodeling. Part II: calvarial bone. Clin Oral Implants Res 2009; 20: 1254-1264.

23) Esteves J, Marcantonio Jr E, de Souza Faloni A, Rocha FR, Marcantonio R, Wilk K, et al. Dynamics of bone healing after osteotomy with piezosurgery or conventional drilling histomorphometrical, immunohistochemical, and molecular analysis. J Transl Med 2013; 11: 221.

24) Zhao D, Witte F, Lu F, Wang J, Li J, Qin L. Biomaterials current status on clinical applications of magnesium-based orthopaedic implants: A review from clinical translational perspective. Biomaterials 2017; 112: 287-302.

25) Windhagen H, Radtke K, Weizbauer A, Diekmann J, Noll $\mathrm{Y}$, Kreimeyer $\mathrm{U}$, et al. Biodegradable magnesium-based screw clinically equivalent to titanium screw in hallux valgus surgery : short term results of the first prospective, randomized, controlled clinical pilot study. Biomed Eng Online 2013; 12: 62 .

26) Jang Y, Collins B, Sankar J, Yun Y. Effect of biologically relevant ions on the corrosion products formed on alloy AZ31B: An improved understanding of magnesium corrosion. Acta Biomater 2013; 9: 8761-8770.

27) Ezechieli M, Ettinger M, König C, Weizbauer A, Helmecke $\mathrm{P}$, Schavan R, et al. Biomechanical characteristics of bioabsorbable magnesium-based (MgYREZr-alloy) interference screws with different threads. Knee Surgery, Sport Traumatol Arthrosc 2016; 24: 3976-3981.

28) Reifenrath J, Angrisani N, Erdmann N, Lucas A, Waizy H, Seitz JM, et al. Degrading magnesium screws ZEK100: Biomechanical testing, degradation analysis and soft-tissue biocompatibility in a rabbit model. Biomed Mater 2013; 8: 045012.

29) Li Z, Gu X, Lou S, Zheng Y. The development of binary MgCa alloys for use as biodegradable materials within bone. Biomaterials 2008; 29: 1329-1344.

30) Rettig R, Virtanen S. Composition of corrosion layers on a magnesium rare-earth alloy in simulated body fluids. $\mathrm{J}$ Biomed Mater Res A 2009; 88: 359-369.

31) Cianferotti L, Gomes AR, Fabbri S, Tanini A, Brandi ML. The calcium-sensing receptor in bone metabolism: from bench to bedside and back. Osteoporos Int 2015; 26: 2055-2071.

32) Razzaghi R, Pidar F, Momen-Heravi M, Bahmani F, Akbari $\mathrm{H}$, Asemi Z. Magnesium supplementation and the effects on wound healing and metabolic status in patients with diabetic 
foot ulcer: A randomized, double-blind, placebo-controlled trial. Biol Trace Elem Res 2018; 181: 207-215.

33) Coger V, Million N, Rehbock C, Sures B, Nachev M, Barcikowski S, et al. Tissue concentrations of zinc, iron, copper, and magnesium during the phases of full thickness wound healing in a rodent model. Biol Trace Elem Res 2019; 191: 167-176.

34) Witte F. Reprint of: The history of biodegradable magnesium implants: A review. Acta Biomater 2015; 23: S28-40.

35) Ved N, Haller JO. Periosteal reaction with normal-appearing underlying bone: a child abuse mimicker. Emerg Radiol 2002; 9: 278-282.

36) Ketteler M, Westenfeld R, Schlieper G. Pathogenesis of vascular calcification in dialysis patients. Clin Exp Nephrol 2005; 265-270.

37) Chen NX, Moe SM. Pathophysiology of vascular calcification. Curr Osteoporos Rep 2015; 13: 372-380.

38) Li Z, Shizhao S, Chen M, Fahlman BD, Debao Liu, Bi H. In vitro and in vivo corrosion, mechanical properties and biocompatibility evaluation of $\mathrm{MgF} 2$-coated $\mathrm{Mg}$ - $\mathrm{Zn}-\mathrm{Zr}$ alloy as cancellous screws. Mater Sci Eng C 2017; 75: 1268-1280.

39) Lee JW, Han HS, Han KJ, Park J, Jeon H, Ok M-R, et al. Long-term clinical study and multiscale analysis of in vivo biodegradation mechanism of $\mathrm{Mg}$ alloy. Proc Natl Acad Sci 2016; 113: 716-721.

40) Zhang L, Pei J, Wang H, Shi Y, Niu J, Yuan F, et al. Facile preparation of poly(lactic acid)/brushite bilayer coating on biodegradable magnesium alloys with multiple functionalities for orthopedic application. ACS Appl Mater Interfaces 2017; 9: 9437-9448.

41) Prasad K, Bazaka O, Chua M, Rochford M, Fedrick L, Spoor $\mathrm{J}$, et al. Metallic biomaterials: Current challenges and opportunities. Materials (Basel) 2017; 10: 884.

42) Naujokat H, Seitz JM, Açil Y, Damm T, Möller I, Gülses A, et al. Osteosynthesis of a cranio-osteoplasty with a biodegradable magnesium plate system in miniature pigs. Acta Biomater 2017; 62: 434-445.

43) Mao L, shen L, Chen J, Zhang X, Kwak M, Wu Y, et al. A promising biodegradable magnesium alloy suitable for clinical vascular stent application. Sci Rep 2017; 7: 46343. 